\title{
Thoracic Chordoma Misdiagnosed as Primary Adenocarcinoma of the Mediastinum
}

\author{
Seung Keun Yoon, M.D., Mi Hyoung Moon, M.D., Ph.D., Seok Whan Moon, M.D., Ph.D. \\ Department of Thoracic and Cardiovascular Surgery, Seoul St. Mary's Hospital, College of Medicine, The Catholic University of Korea, Seoul, Korea
}

\section{ARTICLE INFO}

Received May 6, 2020

Revised June 30, 2020

Accepted July 24, 2020

Corresponding author

Seok Whan Moon

Tel $82-2-2258-6410$

Fax 82-2-594-8644

E-mail swmoon@catholic.ac.kr

ORCID

https://orcid.org/0000-0003-3348-6011

\begin{abstract}
Chordoma is a rare malignant bone tumor originating from the embryonic notochord. Herein, we present a case of thoracic chordoma located at T3-T5 that was misdiagnosed as primary mediastinal adenocarcinoma. The patient underwent neoadjuvant chemoradiation and the disease showed little response. Due to vertebral body invasion, we performed en bloc mass removal and partial corpectomy (T4-5) in collaboration with orthopedic surgeons.
\end{abstract}

Keywords: Chordoma, Mediastinal neoplasms

\section{Case report}

A 52-year-old women was admitted to the hospital for dysphagia of 3 months' duration. Three years earlier, she received a diagnosis of an esophageal submucosal tumor in the mediastinum from another hospital, and had regularly received outpatient follow-up since then. Computed tomography (CT) of the chest revealed a well-defined mass $(30 \mathrm{~mm} \times 19 \mathrm{~mm})$ in the mediastinum at the T4-5 level, with features suspicious of bony erosion (Fig. 1A). Endoscopic ultrasound-guided fine-needle aspiration biopsy of the mass confirmed poorly differentiated carcinoma, favoring adenocarcinoma. Positron emission tomographyCT images disclosed mild heterogenous fluorodeoxyglucose uptake (maximum standardized uptake value $=3.7$ ), with no evidence of distant metastasis (Fig. 1B). Upon review of the findings, a multidisciplinary team decided on neoadjuvant concurrent chemoradiation for this presumptive primary mediastinal adenocarcinoma. The patient received 5 cycles of paclitaxel and cisplatin with a total irradiation dose of $46 \mathrm{~Gy}$, after which her disease remained stable. We then issued a referral for surgical treatment.

Subsequent magnetic resonance imaging (MRI) revealed a heterogeneously enhancing mass $(33 \mathrm{~mm} \times 32 \mathrm{~mm} \times 18$ $\mathrm{mm}$ ) of intermediate signal intensity on both T1- and T2weighted studies of the mediastinum. The mass abutted the trachea and aortic arch, without definitive invasion, and was indistinguishable from the esophagus. Lying in close juxtaposition to the T3-5 vertebrae, it invaded the body of T5. We had procured orthopedic surgical assistance, and the procedure began with video-assisted thoracotomy at the fourth intercostal space. The mass was located between the aortic arch and main pulmonary artery (Supplementary Video 1). The ligamentum arteriosum was clipped and divided, and the mass loosely abutted the esophagus. Feeding vessels originating from the intercostal arteries were also clipped and divided. The tumor base encroached the vertebral body; thus, partial corpectomy (T45) was performed by the orthopedic surgeons for en bloc removal.

The patient's recovery was uneventful, with no residual neural deficits. Discharge was on postoperative day 7. The final pathologic diagnosis was chordoma, with clear resection margins (Fig. 2). The tumor cells displayed strong immunostaining for epithelial membrane antigen, pancytokeratin, and phosphatase and tensin homolog protein. A study for another cytokeratin, S-100 immunostaining, was negative. The multidisciplinary team chose to conduct close follow-up without adjuvant treatment, and follow-up MRI after 1 year demonstrated no evidence of recurrence.

This study was reviewed and approved by the Institutional Review Board of Seoul St. Mary's Hospital and the 

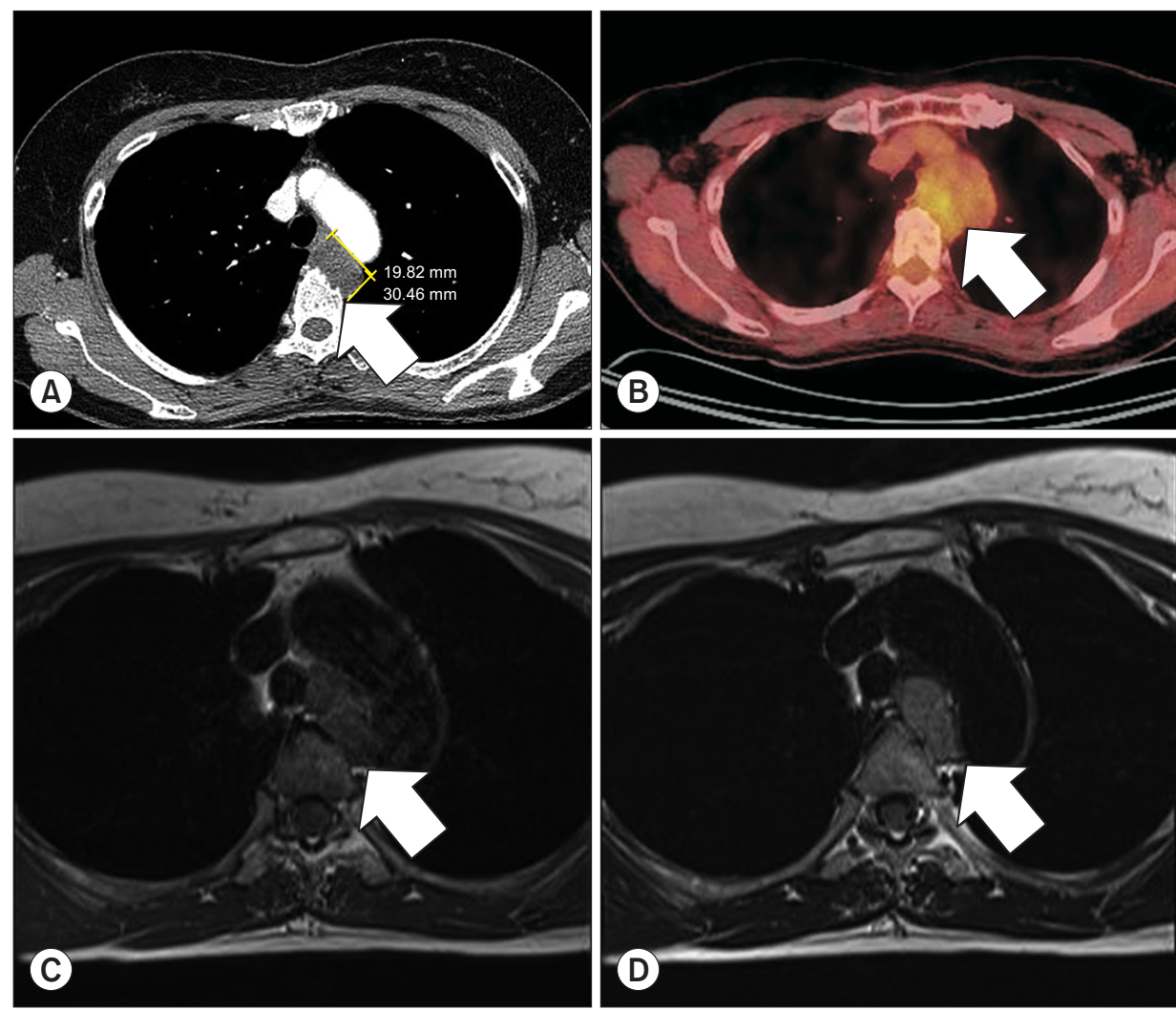

Fig. 1. (A) Initial CT of the chest showing a 30-mm ovoid, well-defined mass (arrow) in the mediastinum (T4-5 level), abutting the wall of the esophagus, with some bony erosion of the adjacent thoracic spine (low attenuation [40 Hounsfield units] on contrast-enhanced CT suggesting neurogenic tumor, bronchogenic cyst, or remotely submucosal esophageal tumor); (B) initial positron emission tomography/ CT imaging of the low-density mass with mild heterogeneous fluorodeoxyglucose uptake (maximum standardized uptake value $=3.7$ ); and (C, D) preoperative magnetic resonance of the stable mass ( $33 \mathrm{~mm} \times 32 \mathrm{~mm} \times 18 \mathrm{~mm}$ ) in the thoracic spine after neoadjuvant chemoradiation; note the heterogeneous enhancement (arrow) with intermediate signal intensity on both T1- and T2-weighted studies, the mass abutting the trachea and the aortic arch (no definitive invasion) that was indistinguishable from the esophagus, and the eroded body of T5 (alongside the T3-5 vertebrae). CT, computed tomography.

requirement for informed consent was waived (IRB no., KC20ZISI0638).

\section{Discussion}

Chordoma is a rare malignant bone tumor that likely arises from the embryonic notochord [1]. Its embryological origins may thus explain why the axial skeleton (primarily the spine or the base of the skull) is commonly affected. However, the incidence and bodily distribution of chordoma are subject to debate. The US Surveillance, Epidemiology, and End Results registry lists an incidence of 0.8 per million persons per year, whereas in European and Taiwanese population-based studies, the respective rates are 0.18 and 0.52 per million persons per year. Because the bias in population-based registry studies is inherently low, these disparities likely stem from genetic differences. Most chor- domas are sacrococcygeal (50\%-55\%) in location, followed by the craniooccipital region (25\%-30\%), cervical spine (8\%), and lumbar spine (5\%). Only $3 \%$ of all chordomas develop in the thoracic spine [2].

Chordoma is considered a low-to-intermediate grade and slow-growing (albeit relentless) malignancy that recurs locally and metastasizes late. According to the World Health Organization classification, chondroid chordoma refers to chordoma in which a large area of the matrix mimics hyaline cartilaginous tumors. Differentiated chordoma has a biphasic appearance, characterized by conventional chordoma and high-grade sarcoma [3]. The mass in this case did not show any evidence of differentiation, thus the diagnosis was chordoma not otherwise specified. Its strong tendency to recur at surgical sites and the fair chance of refractory behavior to therapeutic modalities (radiation, systemic agents) may prove lethal [4]. Thoracic chordoma pre- 

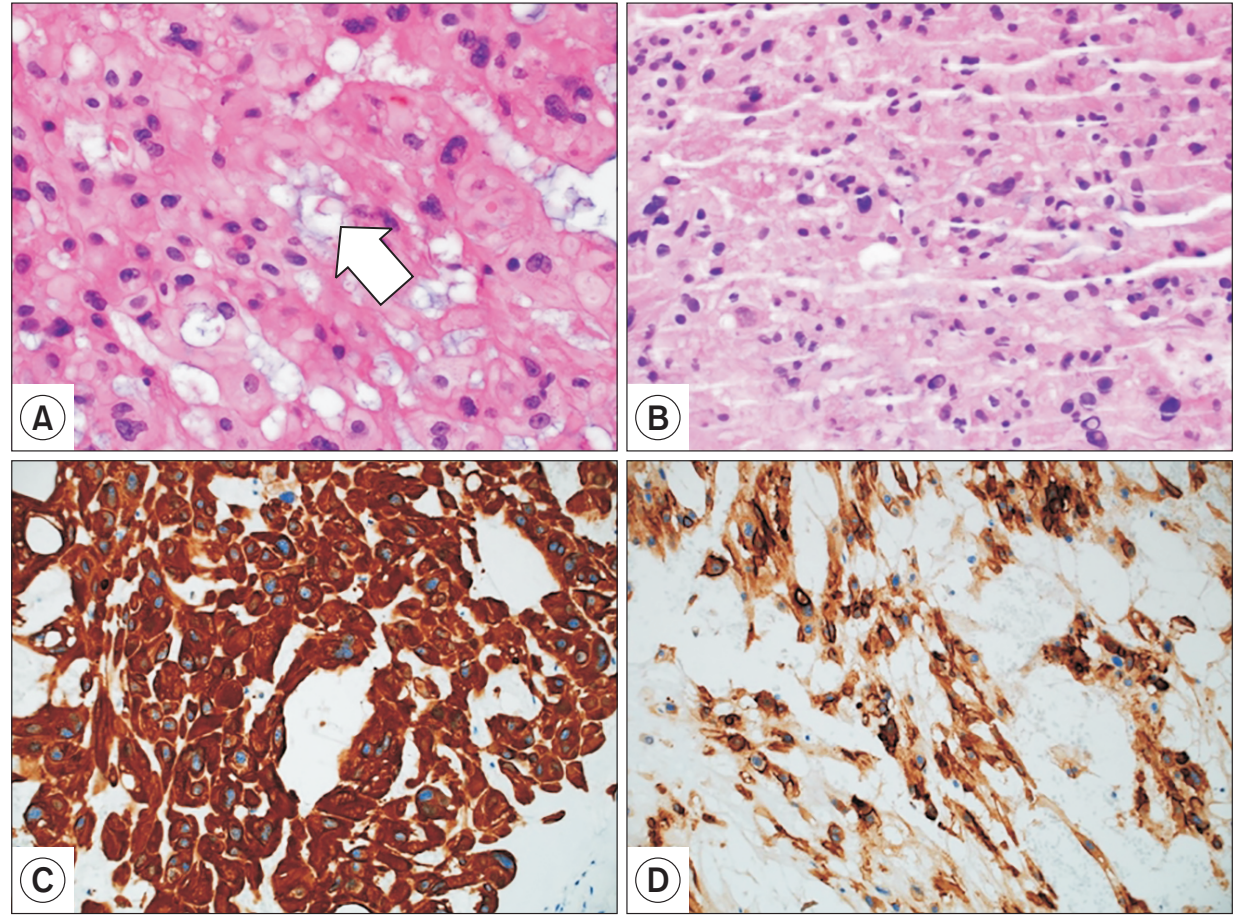

Fig. 2. (A) Area of tumor populated by large cells with abundant, bubbly cytoplasm (arrow), so-called physaliferous cells, typical of chordomas (H\&E, ×200); (B) endoscopic ultrasound-guided fine needle aspiration biopsy failed to provide a definitive diagnosis $(H \& E, \times 200)$, although pancytokeratin positivity and mucin-filled vacuoles suggested adenocarcinoma $(\mathrm{H} \& \mathrm{E}, \times 200)$; (C) pancytokeratin positivity; and (D) epithelial membrane antigen positivity. dominantly occurs at the upper spinal levels, usually $\mathrm{T} 3$ and T4. Patients may or may not be symptomatic, depending on whether the contiguous anatomy (i.e., trachea, esophagus, or neural structures) is compressed or involved. Vertebral body destruction may be evident radiographically. CT and MRI studies are imperative for evaluating the full extent of disease.

Surgery is the principal treatment of chordoma, and en bloc resection should be performed if feasible to achieve tumor-free margins and prevent dissemination [5]. Thoracic chordomas are difficult to resect entirely, although a thorough preoperative work-up and careful evaluation of the extension of the tumor into the spinal canal will guide the surgical plan. Orthopedic or neurosurgical expertise may increase the chances of $\mathrm{R} 0$ resection and cure. The expected 5-year recurrence-free survival rate after R0 resection is $>50 \%$, but reported 5 -year survival rates vary greatly, ranging from $10 \%$ to $70 \%$ [6]. Adjuvant radiotherapy for R0-resected disease is controversial. Monitoring patients for at least 15 years is advised as surveillance for potential recurrence [7].

In adults, posterior mediastinal masses are largely benign, with the majority (approximately 60\%) being neurogenic tumors. Those that are not benign are surgically problematic. Herein, we have described a patient whose mediastinal mass was unusual, mistaken for primary adenocarcinoma of the mediastinum. Neoadjuvant chemoradiation was subsequently administered. Lee et al. [5] previously presented a case of posterior mediastinal chordoma that was also incorrectly interpreted as a submucosal esophageal tumor prior to surgery; and Kim et al. [8] reported a large posterior mediastinal mass thought to be schwannoma, prompting a 2-stage operation. Tissue diagnosis is certainly not mandatory in advance of complete surgical resection. However, if imaging or clinical features are nebulous, or if extensive resection and reconstruction are at hand, the need for tissue diagnosis becomes more pressing. Even so, histologic findings may remain elusive, as in this patient.

In conclusion, a mediastinal mass with locally invasive features requires careful evaluation by a multidisciplinary team. Thoracic chordomas are exceedingly rare malignant tumors originating from notochord remnants. Aggressive treatment through en bloc resection and adjuvant radiotherapy (as needed) may improve patient survival.

\section{Conflict of interest}

No potential conflict of interest relevant to this article was reported. 


\section{ORCID}

Seung Keun Yoon: https://orcid.org/0000-0002-2609-2148 Mi Hyoung Moon: https://orcid.org/0000-0003-2799-4570

Seok Whan Moon: https://orcid.org/0000-0003-3348-6011

\section{Supplementary materials}

Supplementary materials can be found via https://doi. org/10.5090/jcs.20.030. Supplementary Video 1. VATS-assisted thoracotomy and mediastinal mass \& vertebral body en bloc resection.

\section{References}

1. Bakker SH, Jacobs WCH, Pondaag W, et al. Chordoma: a systematic review of the epidemiology and clinical prognostic factors predicting progression-free and overall survival. Eur Spine J 2018;27:3043-58.

2. Maesen F, Baur C, Lamers J, Versteege C, Willighagen R. Chordoma of the thorax. Eur J Respir Dis 1986;68:68-72.

3. Rosenberg AE, Brown GA, Bhan AK, Lee JM. Chondroid chordoma: a variant of chordoma: a morphologic and immunohistochemical study. Am J Clin Pathol 1994;101:36-41.

4. Rahman AM, Farahat IG, Ali WA, Mansour KA. Giant mediastinal chordoma. Ann Thorac Surg 2002;73:1952-4.

5. Lee SH, Choi YS, Kim K, Kim J, Shim Y. Posterior mediastinal chordoma: 1 case report. Korean J Thorac Cardiovasc Surg 2003;36: 707-10.

6. Rena O, Davoli F, Allegra G, Casadio C, Turello D. Giant chordoma of the upper thoracic spine with mediastinal involvement: a surgical challenge. Asian Spine J 2014;8:353-6.

7. Stacchiotti S, Sommer J; Chordoma Global Consensus Group. Building a global consensus approach to chordoma: a position paper from the medical and patient community. Lancet Oncol 2015;16:e71-83.

8. Kim S, Yoo B, Baaj A, Little AG. Resection of a posterior mediastinal mass: lessons learned from a failed exploration for presumed schwannoma. J Thorac Cardiovasc Surg 2016;152:e75-7. 